\title{
Acute Intermittent Porphyria - an Unexpected Association in a Patient with Newly Diagnosed Crohn's Disease
}

\author{
Călin Mitre $^{1,2}$, Voicu Mercea ${ }^{1,2}$, Ioana Maria Copaciu ${ }^{2}$, Andrei Otto Mitre $^{1}$, Caius Breazu ${ }^{1,2}$, Adina Hadade ${ }^{2}$
}

1) Iuliu Hatieganu University

of Medicine and Pharmacy,

Cluj-Napoca

2) Regional Institute of

Gastroenterology and

Hepatology, Cluj-Napoca,

Romania

\footnotetext{
Address for correspondence:

Voicu Mercea

Iuliu Hatieganu University

of Medicine and Pharmacy,

Regional Institute of

Gastroenterology and

Hepatology, Cluj-Napoca,

Romania

Str. Croitorilor 19-21 Cluj-

Napoca cod. 400162, Romania

vmercea@yahoo.com
}

\begin{abstract}
The association of Crohn's disease (CD) with acute intermittent porphyria (AIP), both without a family or personal pathological history, is a very rare clinical possibility. We present the case of a 23 -year-old male diagnosed on the same admission with ileal CD and with an AIP crisis. The diagnosis was challenging as the symptoms overlapped. Crohn's disease was complicated with intestinal occlusion and sepsis; the inflammatory, metabolic and septic changes represented the trigger factor for the first AIP seizure. The neurological symptoms were the key element for AIP diagnosis. The presence of atypical extra-intestinal manifestations in CD patients should raise also the possibility of AIP.
\end{abstract}

Key words: acute intermittent porphyria - Crohn's disease - challenging diagnosis.

Abbreviations: AIP: acute intermittent porphyria; ALA: aminolevulinic acid; CD: Crohn's disease; PBG: porphobilinogen.

\section{INTRODUCTION}

Heme plays a vital part in the synthesis of haemoproteins (such as haemoglobin, cytochromes myoglobin, catalase, peroxidase) involved in oxygen delivery and the redox balance) [1].

Acute intermittent porphyria (AIP) is an autosomal dominant genetic metabolic disease, which causes the reduction with $50 \%$ of the porphobilinogen (PBG) deaminase, which converts porphobilinogen to hydroxymethylbilane [2]. This enzyme deficiency results in accumulation of $\delta$-aminolevulinic acid (ALA) and porphobilinogen (PBG), and the reduction of heme biosynthesis in liver cells. Heme reduction through negative feedback activates ALA-synthetasis, followed by an increase in porphyrins levels (Supplementary Fig. 1).
Alongside with variegata porphyria, hereditary coproporphyria and ALA dehydratase deficiency, acute intermittent porphyria (AIP) is part of a larger group of diseases named acute hepatic porphyrias [3].

Although AIP is rarely seen in clinical practice, with only 2-3 cases out of 100,000 each year, the real incidence of enzymatic changes in apparently healthy patients is much higher [4]. Only $20 \%$ of patients with genetic alterations exhibit clinical symptoms in the presence of a trigger [5].

Acute intermittent porfiria has symptoms from the central and peripheral nervous systems and from the autonomic nervous system [6]. Because of its low incidence and nonspecific symptoms, AIP is usually a diagnostic challenge, being it is initially misdiagnosed.

In addition, the association between an inflammatory disorder of the digestive tract such as Crohn's disease (CD) and the onset of an acute intermittent porphyria crisis, both without a family history, is a very rare clinical possibility, with only four reported cases in the literature, two of them in the English language $[7,8]$.

\section{CASE REPORT}

A 23-year-old male, previously diagnosed with chronic constipation, presented at the Gastroenterology Department for an intestinal subocclusive syndrom (abdominal pain and lack of intestinal transit for three days) asociated with nausea, 
vomiting and fever $\left(38.5^{\circ} \mathrm{C}\right)$. An emergency abdominal CT scan showed the thickened wall of terminal ileum, determined by oedema in the submucosa (Fig 1) and inflammatory lymph nodes. The colonoscopy revealed inflammatory stenosis of the ileo-cecal valve with small ulcerations of the intestinal mucosa. The biopsy was suggestive for CD in acute ulcerative phase. The corticotherapy was initiated, but the patient developed again symptoms of intestinal occlusion. An emergency surgery was performed with ileo-cecal segmental resection and termino-lateral ileo-colonic anastomosis. Histopathological examination confirmed the diagnosis of CD with severe, ulcerative activity (Fig. 2).

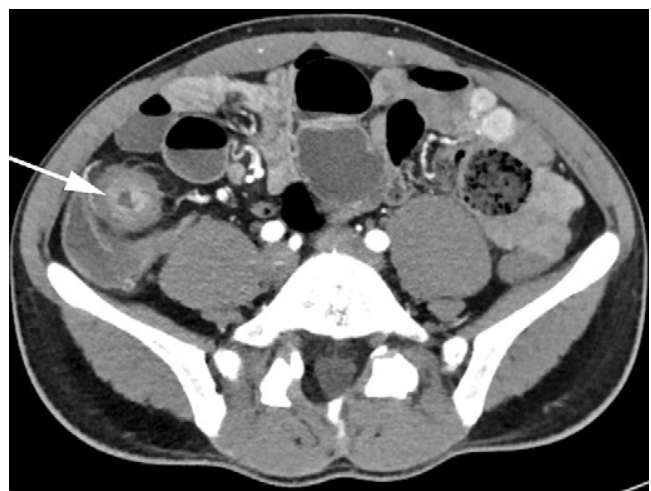

Fig. 1. CT scan: thickened terminal ileum (arrow), determined by oedema in the submucosa.
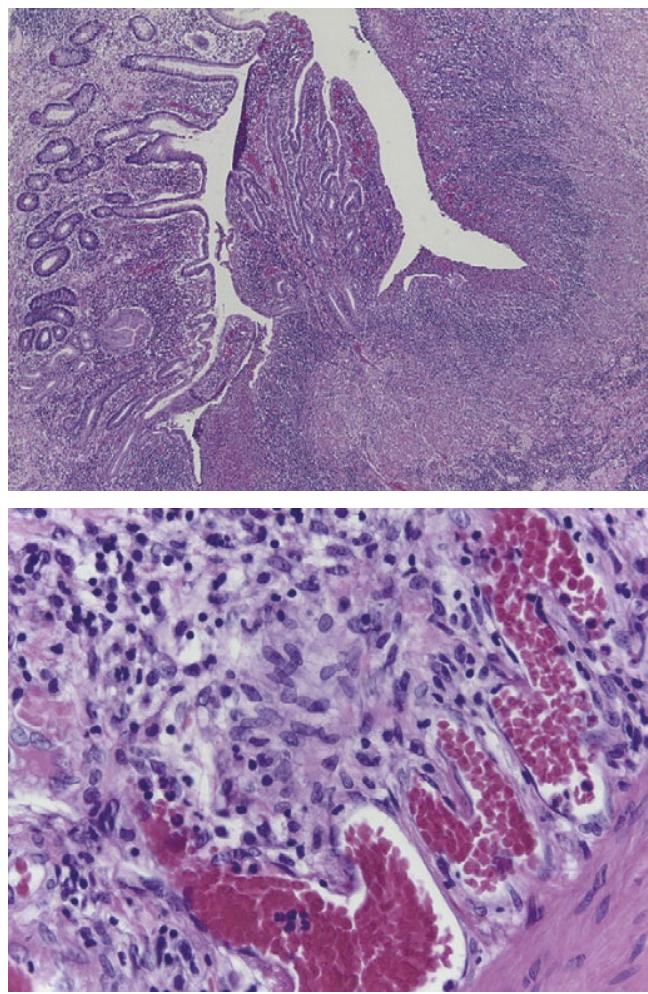

Fig. 2. Histopathology: (A): deep ulcerations and inflammatory cells in the mucosa and submucosa layers (H\&E, 10x); (B): epithelioid granuloma (H\&E, 40x).

Postoperative evolution was complicated by an ileo-colic anastomosis fistula, with secondary stercoral peritonitis. Another surgery was decided and a terminal ileostomy was performed. Peritoneal infection with Enterococcus faecium and
Staphylococcus aureus was documented and antibiotherapy initiated. He presented favourable post operatory evolution, with decreasing inflammatory markers.

At 10 days after the second surgery, the patient exhibited suddenly two generalized tonic-clonic seizures, interpreted in the context of the hyponatraemia that remitted after the hydro-electrolytic imbalance correction. At the same time, the patient experienced intense abdominal pain with nausea and vomiting, persistent headache, behavioural disorder (sleepy, confusion). These symptoms were associated with progressive tetramelic motor dysfunction, predominantly at the inferior limbs, without affecting the diaphragm, but with mild laryngeal paresis, phonation disorders and dysphagia. Contrast cerebral CT scans revealed no pathological modifications. EMG examinations detected mild polyneuropathy, with electrophysiological asymmetric motor and sensory changes at the distal peripheral axonal level. Visual evoked potentials were prolonged bilateral. Polyradiculoneuritis Guillain Barre/Guillain Barre-like syndrome was suspected. However cerebrospinal fluid examination revealed normal cellularity and protein level; antiganglioside antibodies were negative. Virusologic screening for Epstein-Barr virus, cytomegalovirus, herpes simplex 1 and 2 and HIV were negative.

The patient presented arterial tension fluctuations (arterial hyper/hypotension), tachycardia (130 beats/min), polypnea (30 respirations/min), with normal blood oxygenation $(\mathrm{SpO} 2>96 \%)$ interpreted in the context of sepsis. He also presented polyuria $(5.000 \mathrm{~mL} / 24 \mathrm{~h})$ without identification of a common cause, with limited response to liquid restriction and antidiuretic hormone. The desmopressin test was negative, excluding the syndrome of inappropriate $\mathrm{ADH}$ secretion. The patient's urine had a dark-yellow colour occasionally, but did not present the specific colour changes (brown or dark red).

As the neurological symptoms persisted, the patient was admitted to the intensive care unit. The second EMG test indicated a slight worsening of the neurological evolution. An acute motor axonal neuropathy syndrome was suspected and i.v. immunoglobulin treatment was initiated for 5 days, with no significant improvements. Contrast cerebral MRI investigation revealed 4 small demyelination lesions located bilaterally in the deep white matter of the frontal lobe, without areas of acute ischemia or haemorrhagic collections intra or extracerebral white matter. At the spine level, MRI examination revealed minimal degenerating alterations, lacking any pathological significance.

In the absence of a specific sign, we investigated the possibility of an AIP. The urinary $\delta$-aminolevulinic acid, uroporphyrins, porphobilinogen and erythrocyte zinc protoporphyrin were measured two days consecutively, and were found increased: $\delta$-ALA: $11.5 \mathrm{mg} / \mathrm{L}$ (normal range: $5-10 \mathrm{mg} / \mathrm{L})$; uroporphyrins: $93.1 \mu \mathrm{g} / \mathrm{L}(<25 \mu \mathrm{g} / \mathrm{L})$; porphobilinogen: $15.1 \mathrm{mg} / \mathrm{L}(<2 \mathrm{mg} / \mathrm{L})$; erythrocyte zinc protoporphyrin: $5 \mu \mathrm{g} / \mathrm{g} \mathrm{Hb}(0.7-4 \mu \mathrm{g} / \mathrm{g} \mathrm{Hb})$. After six weeks evolution, the final diagnosis of AIP was reached. Nonspecific treatment with carbohydrates (300-400 g/day) was started. The neurological symptoms improved under hypercaloric treatment and kinetotherapy. The patient was safely discharged with minimally neurological impairment. The patient was admitted to a specialised centre for porphyrias 
for further genetical investigations and specific treatment. $\mathrm{He}$ started treatment with Normosang (human menine) $4 \mathrm{mg} /$ $\mathrm{kg} /$ day for 4 days via central catheter, followed by symptoms resolution.

Furthermore, two other family members (brother and sister) have been identified with an enzymatic deficiency that could lead to AIP, but they had no symptoms.

\section{DISCUSSION}

The clinical presentation of the AIP seizures is represented by symptoms predominantly attributed to central, peripheral and autonomic neuropathy, being related to the accumulation of porphyrin precursors. Central neurological symptoms (incidence $40-58 \%$ ) are represented by agitation, psychosis, seizures (10-20\%), posterior reversible encephalopathy syndrome. Peripheral neurological symptoms (incidence 42-68\%) include progressive peripheral neuropathy, muscle weakness-might progress to quadriparesis and respiratory failure-requires mechanical ventilation. Autonomic instability symptoms (incidence $85-95 \%$ ) are represented by abdominal pain, nausea, vomiting, constipation or diarrhoea, hypertension, tachycardia, postural hypotension, urinary incontinence or retention. Additionally, a patient with AIP can also present biochemical abnormalities: hyponatremia, hypomagnesemia, hypochloremia, hypokalemia [9].

The precise mechanism through which these signs and symptoms occur remains unknown [5]. It is suggested that ALA or an ALA metabolite would have a direct neurotoxic effect, by reacting with the $\gamma$-amino butyric acid receptor, altered tryptophan metabolism, or a neural respiratory hemedependent enzymatic deficiency in nerve cells. The damage to peripherical and autonomous nerve axons is more of a degenerative type, rather than of demyelination [10].

Nevertheless, the clinical diagnosis of AIP, especially at first presentation is difficult, because these symptoms are common to other diseases more often present in the clinical practice. The abdominal symptoms of our patient were interpreted in the CD context; the hemodynamic instability and hyponatremia, with subsequently neurological symptoms were attributed to sepsis developed in the evolution of CD. The worsening of peripheral polyneuropathy, not explained by $\mathrm{CD}$, was the key for AIP diagnosis. Interestingly, what lacked in our patient's symptoms and is specific for AIP was the colour modification of the urine (dark brown-red urine). Even though darker, it had not the red-brown hue that is characteristic for porphyria.

In our case the established diagnosis of $\mathrm{CD}$ delayed the AIP diagnosis. The CD complications (lack of food intake for a few days, immunosuppression, occlusive syndrome, sepsis secondary to stercoral peritonitis or the surgical stress by itself) represented most probably the triggers for AIP seizure [8]. Also the inflammation processes of $\mathrm{CD}$, through oxidative stress on the digestive tract, might have stimulated heme oxygenase-1 (HO-1). This enzyme catalyses heme degradation and finally removes feedback inhibition of pre-5-aminolevulinic acid synthase 1 (pre-ALAS1) produced by the heme and thus promotes porphyria $[11,12]$.

Treatment of our patient included antibiotherapy, hydroelectrolytic and acido-basic correction, parenteral and later enteral nutrition. For the AIP diagnosis, the patient received the recommended dose of 300-400 g/ day carbohydrates. The intravenous intake of glucose, carbohydrates or gluconeogenic aminoacids attenuates the hunger state, thus reducing ALAS1 activity and stimulating the heme negative feedback on its synthesis $[10,13]$.

The lack of specificity of the clinical picture from AIP as well as the lack of experience with such cases, due to a lower clinical incidence, can lead to a delay in reaching the correct diagnosis. Many of the AIP symptoms are also present in other abdominal or neurological diseases, which complicates the diagnosis. It is recommended to perform a simple screening WatsonSchwartz urinary test at the lowest porphyria suspicion, usually represented by the onset of abdominal pain accompanied by central and peripheral neurological alterations, without any clear cause and non-responding to treatment.

The presence of porphyritic precursors ALA and PGB in the urine represents the diagnostic test. Also, their presence in the urine will change its colour at light exposure from yellow to dark red or brown [9]. Identifying the triggers of AIP will also raise the degree of suspicion for this pathology.

\section{CONCLUSIONS}

A first episode of $\mathrm{CD}$, complicated with septic surgical pathology represented, through their inflammatory, metabolic and septic changes, the trigger factor for the first AIP seizure in our patient. The association of CD with AIP is very rare, but should be considered in patients with complicated inflammatory bowel disease associating neurological and or urinary symptoms.

\section{Conflict of interest: None to declare.}

Authors' contribution: A.O.M., V.M., A.H.: drafted the paper; C.M, I.M.C., A.H., C.B.: managed the patient. C.M.: critically revised of the final version of the manuscript. All authors approved the final version of the manuscript.

Supplementary material: To access the supplementary material visit the online version of the J Gastrointestin Liver Dis at http://dx.doi. org/10.15403/jgld-547

\section{REFERENCES}

1. Fu Y, Jia J, Yue L, et al. Systematically Analyzing the Pathogenic Variations for Acute Intermittent Porphyria. Front Pharmacol 2019;10:1018. doi:10.3389/fphar.2019.01018

2. Gill R, Kolstoe SE, Mohammed F, et al. Structure of human porphobilinogen deaminase at $2.8 \mathrm{~A}$ : the molecular basis of acute intermittent porphyria. Biochem J 2009;420:17-25. doi:10.1042/ BJ20082077

3. Stölzel U, Doss MO, Schuppan D. Clinical Guide and Update on Porphyrias. Gastroenterology 2019;157:365-381.e4. doi:10.1053/j. gastro.2019.04.050

4. González Estrada A, García-Morillo S, Gómez Morales L, Stiefel GarcíaJunco P. Chronic Elevation of Liver Enzymes in Acute Intermittent Porphyria Initially Misdiagnosed as Autoimmune Hepatitis. Int J Hepatol 2011;2011:392049. doi:10.4061/2011/392049 
5. Anderson KE, Bloomer JR, Bonkovsky HL, et al. Recommendations for the diagnosis and treatment of the acute porphyrias. Ann Intern Med 2005;142:439-450. doi:10.7326/0003-4819-142-6-20050315000010

6. O’Malley R, Rao G, Stein P, Bandmann O. Porphyria: often discussed but too often missed. Pract Neurol 2018;18:352-358. doi:10.1136/ practneurol-2017-001878

7. Beckh K, Sieg I, Stellwaag M, Blanke H, Arnold R, Doss M. Coincidence of acute intermittent porphyria and Crohn's disease. Dtsch Med Wochenschr 1990;115:817-820. doi:10.1055/s-2008-1065086

8. Sieg I, Beckh K, Kersten U, Doss MO. Manifestation of acute intermittent porphyria in patients with chronic inflammatory bowel disease. Z Gastroenterol 1991;29:602-605.

9. Menegueti MG, Gil Cezar AT, Casarini KA, et al. Acute Intermittent Porphyria Associated with Respiratory Failure: A
Multidisciplinary Approach. Crit Care Res Pract 2011;2011:283690. doi: $10.1155 / 2011 / 283690$

10. Naithani BK, Shah SB, Bhargava AK, Batra V. Anesthesia for hemicolectomy in a known porphyric with cecal malignancy. Saudi J Anaesth 2015;9:82-85. doi:10.4103/1658-354X.146320

11. Yasuda M, Erwin AL, Liu LU, et al. Liver transplantation for acute intermittent porphyria: biochemical and pathologic studies of the explanted liver. Mol Med 2015;21:487-495. doi:10.2119/molmed.2015.00099

12. Doberer D, Haschemi A, Andreas M, et al. Haem arginate infusion stimulates haem oxygenase-1 expression in healthy subjects. Br J Pharmacol 2010;161:1751-1762. doi:10.1111/j.14765381.2010.00990.x

13. Handschin C, Lin J, Rhee J, et al. Nutritional regulation of hepatic heme biosynthesis and porphyria through PGC-1alpha. Cell 2005;122:505515. doi:10.1016/j.cell.2005.06.040 


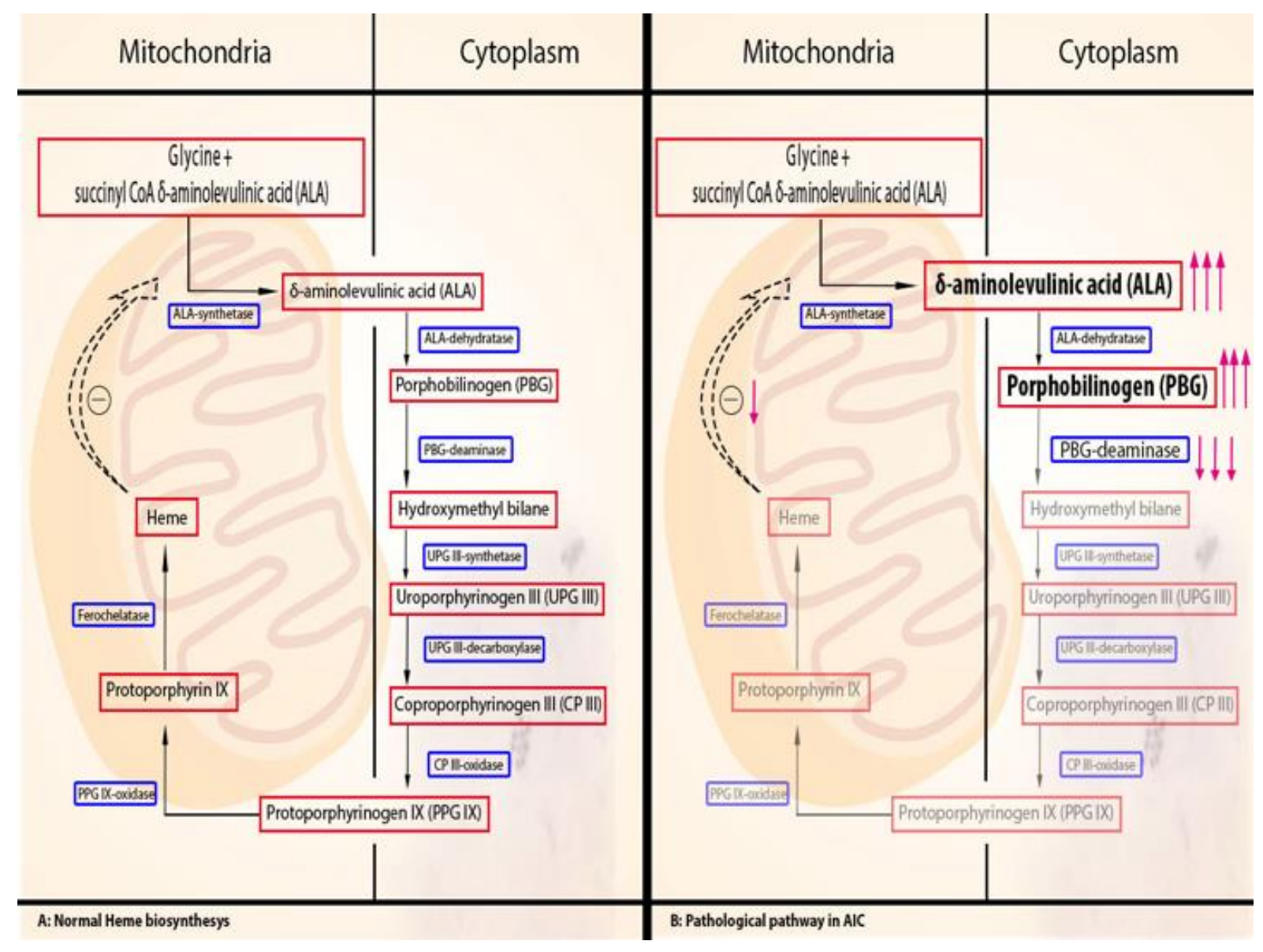

Supplementary Fig 1. (A) Normal heme biosynthesis pathway; (B) Pathological pathway in acute intermittent porphyria 